\title{
THE PHYSICS OF FLARE STARS
}

\author{
DAVID S. EVANS*
}

McDonald Observatory and Dept. of Astronomy, University of Texas at Austin, Austin, Tex., U.S.A.

\begin{abstract}
This paper reports the researches of the Texas flare star group. High time resolution and simultaneous photometric, spectroscopic and, if possible, radio observations of flaring stars are essential. Fast and slow flares are distinguished and their distinct optical, spectroscopic, and probably, radio effects are noted. The starspot model satisfactorily accounts for many optical and spectroscopic flare star properties, including the precursor phenomenon and quasi periodicity of flaring found by some observers. The basic mechanism of flares is thought to be magnetic field collapse on a scale vastly greater than in the Sun, but further observations are essential, even though there are theoretical grounds for support. No analogue of the solar cycle has yet been detected. The problem of duplicity remains a puzzle.
\end{abstract}

I must make it clear that I speak, not only on my own behalf but also on behalf of my students and colleagues who have so actively pursued the flare star programme at Texas. They are, Dr Bernard W. Bopp, Dr Thomas J. Moffett, and Mr Steven S. Vogt. At Texas, greatly inspired by Dr R. Edward Nather, we have pursued a number of different types of high speed photometric programme, including one on flare stars. Since the term has been rather loosely used, I would define high speed photometry as an activity in which observations are obtained with a time resolution of one second or better. According to the strict rules of grammar it might be called second rate astronomy, but it has proved to be anything but that. One must insist on the

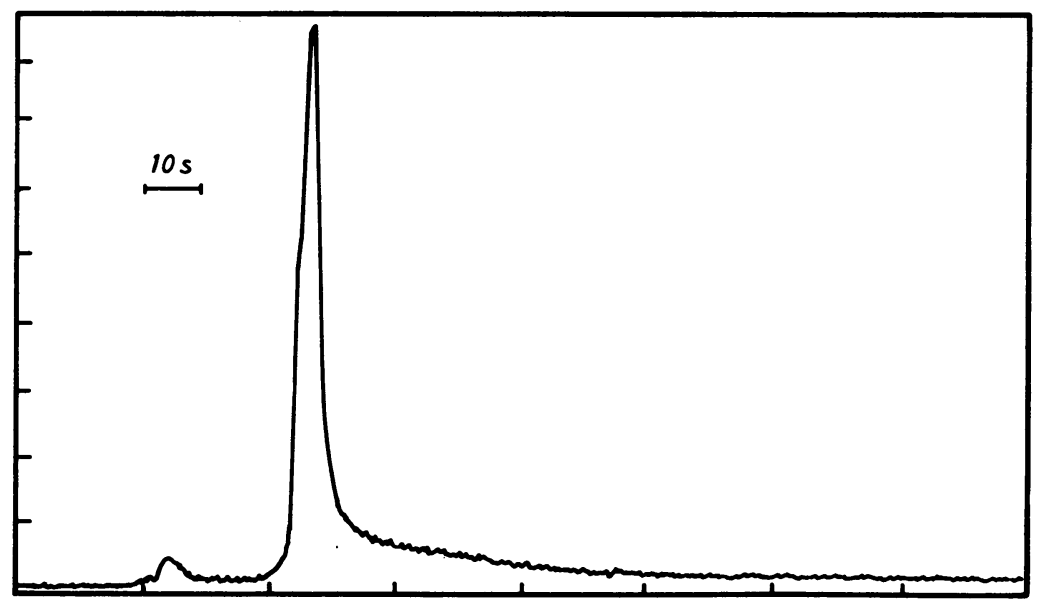

Fig. 1. A typical high speed flare record after Moffett (1972). The original is composed of discrete points observed at $1 \mathrm{~s}$ intervals. The significant elements in the figure are (i) the spike flare lasting less than $10 \mathrm{~s}$, (ii) the slow flare component, (iii) the precursor which precedes the main flare.

* Summer Visiting Professor, Geneva Observatory, Switzerland. 
definition since some authors have described their work as 'High speed' when, in fact, they are talking about observations on a scale of minutes. From the sample flare curve shown in Figure 1, taken from Moffett (1972), one can see that an entire drama can be enacted and finished within a minute or less, and that, as Moffett so rightly insists, without adequate time resolution one can lose most of the story. One sometimes sees in the literature representations of flares in which observational points spaced at a minute or so have been joined by a smooth curve. It is not difficult to see how much such a representation may have lost in comparison with the real course of action.

In much of our work we have tried to obtain simultaneous photometric and spectroscopic observations. Many of the more than 400 photometric flare observations obtained by Moffett (1974) have been paralleled spectroscopically by Bopp, achieving in many cases a time resolution of a few tenths of a minute. This work has brought considerable insight (Bopp and Moffett, 1973) and we believe that we now know how to read the kind of curve shown in the figure. It shows several different elements which combine to form what Moffett calls a 'flare event'. The photometric curve falls into two parts - a 'spike flare' of very high peak intensity and short duration, which is superposed on a slow variation which may have a time scale of a minute or more. From the work of Bopp and Moffett we know that the spike flare will have a continuous emission spectrum, whereas the slow flare will show no continuum but enhanced Balmer and ionized calcium emission lines. The slow phase represents the injection into a small atmospheric volume of a relatively large amount of energy, but at not so high a rate that it cannot be dissipated in ordinary recombination processes. During the spike phase the rate of energy injection is so high that a continuum and shock wave are generated. If this tentative picture, due to Moffett, is correct, we should expect radio observations of flare phenomena to be associated with optical flares only of the spike type. The delay between optical and radio incidents remarked by Lovell in his cooperative programmes with Chugainov et al. (1971) then finds a natural explanation as the travel time of a shock wave from the photospheric level to the level at which the appropriate gyrofrequency can be excited.

At Texas we have spent a good deal of effort in the observation of flare stars outside flare activity, and the evaluation of the results in terms of star spots, an idea revived in modern times by Kron (1952) in connection with observations of YY Gem, not then known to be a flare star (see Moffett and Bopp, 1971). This has since been discussed in varying detail by a variety of authors, including Chugainov $(1966,1973)$, Torres and Ferraz Mello (1973), Vogt (1974), Bopp and Evans (1973). I became interested in the idea, apart from a superficial aside (Evans, 1959, 1964) following a conversation with Krzeminski (Evans, 1971). The crucial consideration which excludes other possibilities, and clinches the conclusion that we are dealing with phenomena on the stellar surfaces, is the fact that whereas in YY Gem, CC Eri and other stars, the photometric period is closely equal to the spectroscopic period, in BY Dra it is not. (Bopp and Evans, 1973). Torres (1972) seems to have been the first to realise that all $\mathrm{M}$ dwarfs which show photometric spot phenomena are flare stars. 
For our part we would add the requirement that they show spectra with the Balmer lines in emission, but it may be that this is an invariable accompaniment in any case.

Photometric observations by Masani et al. (1955), Chugainov, (1961, 1966, 1973), Krzeminski and Kraft (1967), Krzeminski (1969), Moffett (1973, 1974), Torres and Ferraz Mello (1973), Torres et al. (1972), Vogt (1974) and myself, all support the thought that star spots must be long lived, with life times of months or years. Also, that they must occupy a considerable fraction of the visible disk of a star, possibly as much as $20 \%$. They must also, in general be darker and cooler than the photosphere, though it will require very precise photometry to define exactly how much cooler. Completely black spots would, of course, be compatible with the first crude impression that in these stars the photometric variations are not accompanied by colour changes. Certainly they are not large, but recent extremely accurate photometric observations by Vogt of BY Dra, in which periodic colour changes of a few thousandths of a magnitude have been detected (1974) show what can be done. I think that this work will demonstrate that when a star spot disappears, as has recently happened on this star, it may leave behind a clutter of plages in the same general area whose photometric effects will be detectable by high quality observations. In any discussion of such subtle results meticulous attention must be paid to problems of the band passes used. We may have to consider other than simple black body temperature effects. For example, a great enhancement of the $\mathrm{TiO}$ bands consequent upon a rather slight temperature change might just encroach on the wing of the $B$-filter band pass and produce a change greater than simple black body theory would lead one to expect. I am indebted to M. Grenon of the Geneva Observatory for illuminating discussions of this point.

The extent of the spot or active area may be approached in another way, as has been shown by the work of Bopp (1974b) on YY Gem. When a flare occurs, the spectroscopic data show which component of the binary is flaring. If all the flares within an interval of a few days are associated with the same active or spot area, it becomes possible to set longitude limits on this, and these turn out to be of the same order as those provided independently by analysis of the out of flare periodic photometric variations. In the same paper Bopp also demonstrates the rotational modulation of emission line strengths outside flares, and this reinforces still further the concept of a large active or spot area on the star surface being carried around by rotation.

In the case of those binary flare stars of low eccentricity we can assume that the stars are rotationally locked, but this is not the case for BY.Dra where the orbit is very eccentric and the two periods differ (Bopp and Evans, 1973).

It is a mistake, I think, to suppose that the Sun is a good guide to the behaviour of spotted flare stars, for it seems clear that the Sun is a feeble example of the breed. Stellar flares are much more energetic than solar; star spots are much bigger and longer lived than sunspots. If the basic mechanism of flare generation is the same, e.g. the collapse of a magnetic field, both may be explicable if the star magnetic fields are much larger. The discussion of this point by Bopp and Evans (1973) needs revision owing to the inadvertent omission of a numerical factor, and it is clear that we may 
have to think in terms of fields of the order of $50000 \mathrm{G}$. Mullan (1974) has considered a convection model for these, surely highly convective, stars, and believes that fields of this order are plausible. Flare stars all appear to be rather rapid rotators, with equatorial velocities in the range from $5 \mathrm{~km} \mathrm{~s}^{-1}$ to $40 \mathrm{~km} \mathrm{~s}^{-1}$, and, as has been pointed out in conversation by Skumanich, there may be merit in the assumption of an empirical correlation between rotation velocity and magnetic field intensity.

Some workers have addressed themselves particularly to questions of rates of flare incidence and recurrence. These important problems are rendered difficult by problems of observational limitations and problems of semantics. What exactly is a flare? For Moffett a flare event may include several separate flares, the later ones, through involvement with the earlier ones, possibly showing on the photometric record as quite inconspicuous spikes or bulges. At the time of writing there are under analysis considerable series of simultaneous optical observations from McDonald and radio observations from Arecibo. It is entirely premature to comment, but one has the impression that it is easier in some ways to recognise a complex event as a sequence of individual flares on the radio record than it may be on the visual record. If this first snap judgement is correct it conforms to the notion that incidents corresponding to slow flares will not be present on the radio record. On the optical record we need the high time resolution before we can begin to attach significance to the notion of some kind of unit flare which occurs at a variety of strengths.

Flares may and do occur in groups. Are they causally connected? I understand that this will be contested in some quarters. However, the final element on my figure is a small flare preceding a larger one, and this we call a 'precursor'. The view that this is in no way a trigger for the later flare may be tenable, but, true or not, our observers have habitually used the indication of a precursor on the photometric record to alert the spectroscopic observer. The hypothesis may be invalid, but by adopting it we have many many flare spectra. The interval between precursor and main flare is not constant. Our working hypothesis is that the active area, a large spot or spot group, is outlined and covered irregularly by plages which are the source of the quiescent emission line spectrum. Flares may occur predominantly around the edges of the active region, generating shocks in the atmosphere which trigger other flares around the spot. (Moffett, 1973). We have, incidentally, not found it possible to sustain the idea that flares on one star of a binary may trigger flares on the other with a delay time corresponding to light or particle travel.

Efforts have been made to pick up periodicity in the occurrence of flares as the result of stellar rotation. Now very subtle methods are available which will detect periodicities in all sorts of data, but it is as well to remember that these methods will pick up periodicities which got there, not only because of real rhythms in the objects studied but also because of artificially imposed periods due to extraneous circumstances. In a case which has nothing to do with flare stars, a colleague of mine was delighted to derive a periodicity of 29.53 days evidently due to the scheduling practices of the observatory in question. An example which is in the flare literature, 
giving a period of $23 \mathrm{~h}$ and 56 min must have got in there in the same sort of way. Some workers have found indications of periodicity, some not. This situation finds a ready explanation if the active area has a considerable longitude range. Indeed, if one cares to refer back to Bopp's work on YY Gem, one can see that if he is right, strong periodicities will not normally be found, and if strong periodicities are found, then flares must occur at a definite longitude and not over an area.

Lastly there is the question of an analogue for the sunspot cycle. BY Dra seems to be the only flare star which has been observed for a very long time, and Vogt's (1974) synoptic discussion shows a variation of spottedness from slight in 1954 and 1960 , to 1965 , when there was strong spot activity, which had been markedly reduced by 1969 . There seem to have been variations in extent and longitude but no evidence of periodicity. If we speculate that there may be a relation between average spot life time and the cycle time, then there simply has not been enough time to detect periodicity. We ought to keep some of these stars under continuous surveillance with standard equipment so that a star which shows spots, and presumably hence a propensity to flare, may be the object of concentrated attention.

Our working model of flare star behaviour explains many things but leaves several questions unanswered and demands several observational programmes. We need observational confirmation of high magnetic fields on these stars correlated with spot activity. Are we right in thinking that magnetic collapse is the whole story, or is there some other source, possibly even nuclear, of the astonishing rates of generation of energy which we observe? The observation of lithium by Bopp (1974c) may be significant here. The rapidly rotating convective configuration may be the right kind of dynamo, but is that the whole story? For star spots to last as long as they do must imply a particular kind of surface rotation. One is, perhaps irrelevantly, teminded of Jupiter with its remarkable scheme of high speed surface rotation and a long enduring large spot. What is the role of duplicity in flare stars? Is this very common feature fundamental or an accident? Perhaps we shall be able to test some aspects of this question by comparing flare rates on UV Ceti with components somewhat separated with results obtained with the same equipment near periastron.

Several concepts occur in the literature which we think may be open to doubt. Observations of flares on EQ Peg (Owen et al., 1972) show that both components flare, and not merely the fainter, as do both components of YY Gem. If we look at the kinematics of flare stars we do not find space velocities indicative either of youth or age. One can hardly believe that a configuration like BY Dra can be very old, but there are no kinematical indications of youth about the other flare stars. Perhaps we should beware of previous generalisations about $M$ dwarfs since many of them depend on YY Gem, now shown to be exceptional.

\section{Acknowledgement}

This work has been supported by National Science Foundation Grant GP-41796. 


\section{References}

Bopp, B. W.: 1974a, Monthly Notices Roy. Astron. Soc. 166, 79.

Bopp, B. W.: 1974b, Astrophys. J. 193, 389.

Bopp, B. W.: 1974c, Publ. Astron. Soc. Pacific 86, 281.

Bopp, B. W. and Evans, D. S. : 1973, Monthly Notices Roy. Astron. Soc. 164, 343.

Bopp, B. W. and Moffett, T. J.: 1973, Astrophys. J. 185, 239.

Chugainov, P. F.: 1961, Izv. Krymsk. Astrofiz. Obs. 26, 171.

Chugainov, P. F.: 1966, Info. Bull. Var. Stars No. 122.

Chugainov, P. F.: 1973, Izv. Krymsk. Astrofiz. Obs. 48, 3.

Evans, D. S.: 1959, Monthly Notices Roy. Astron. Soc. 119, 526.

Evans, D. S.: 1964, Monthly Notices Astron. Soc. S. Africa 23, 68.

Evans, D. S.: 1971, Monthly Notices Roy. Astron. Soc. 154, 329.

Kron, G. E.: 1952, Astrophys. J. 115, 301.

Krzeminski, W.: 1969, in S. Kumar (ed.), Low Luminosity Stars, Gordon and Breach, London, p. 57.

Krzeminski, W. and Kraft, R. P.: 1967, Astron. J. 72, 307.

Lovell, B.: 1971, Q. J. Roy. Astron. Soc. 12, 98.

Masani, A., Broglia, P., and Pestarino, E.: 1955, Contr. Oss. Astron. Milano-Merate, Nuov. Ser., No. 59.

Moffett, T. J.: 1972, Nature Phys. Sci. 240, 41.

Moffett, T. J.: 1973, Monthly Notices Roy. Astron. Soc. 164, 11.

Moffett, T. J. : 1974, Astrophys. J. Suppl., in press.

Moffett, T. J. and Bopp, B. W.: 1971, Astrophys. J. Letters 168, L117.

Mullan, D. J.: 1974, Astrophys. J. 192, 149.

Owen, F. N., Bopp, B. W., Moffett, T. J., and Lazor, F. J. : 1972, Astrophys. Letters 10, 37.

Torres, C. A. O.: 1972, in Symposium on Flare Stars, Saõ Jose dos Campos, Brazil, to be published.

Torres, C. A. O. and Ferraz Mello, S.: 1973, Astron. Astrophys. 27, 231.

Torres, C. A. O., Ferraz Mello, S., and Quast, G. R.: 1972, Astrophys. Letters 11, 13.

Vogt, S. S.: 1974, Astrophys. J., in preparation. 